\title{
INVESTIGATION OF QUEUEING NETWORK WITH UNRELIABLE SYSTEMS AND LARGE NUMBER OF MESSAGES
}

\author{
Mikhail Matalytski ${ }^{1}$, Sviataslau Statkevich ${ }^{2}$ \\ ${ }^{I}$ Institute of Mathematics, Czestochowa University of Technology, Poland \\ ${ }^{2}$ Faculty of Mathematics and Computer Science, Grodno State University, Belarus \\ m.matalytski@gmail.com,sstat@grsu.by
}

\begin{abstract}
The Markov network with unreliable queueing systems and a large number of messages is investigated. The service channels of systems are exposed to random failure, besides the time of the proper functionality and the time of reconstruction of each channel of system has the exponential distribution with distinctive parameters. The system of difference-differential equations of Kolmogorov for the states probabilities is compiled. The partial differential equation for the probability density function of the vector of states is deduced. The systems of ordinary differential equations for an average number of messages and serviceable channels of network systems are received.
\end{abstract}

\section{Introduction}

Let us examine the closed exponential queueing network with the $K$ messages of the same type which consist of $n+1$ queueing systems (QS) $S_{1}, S_{2}, \ldots, S_{n}$. The system $S_{i}$ includes $m_{i}$ identical service channels, $i=\overline{1, n}$, and $m_{0}=K$.

Considering that the service channels of the system $S_{0}$ are absolutely reliable and in the other systems $S_{1}, S_{2}, \ldots, S_{n}$ the service channels are exposed to random failure; besides the time of the proper functionality of each $S_{i}$ system's channel has the exponential distribution with the parameter $\beta_{i}, i=\overline{1, n}$. After the breakage, the channel starts to reconstruct immediately. The time of reconstruction also has exponential distribution with the parameter $\gamma_{i}, i=\overline{1, n}$. After servicing in system $S_{i}$ the message immediately transfers into the system $S_{j}$ with probability $p_{i j}$, $i, j=\overline{0, n}, p_{00}=0, \sum_{j=0}^{n} p_{i j}=1, i=\overline{0, n}$. The matrix $P=\left\|p_{i j}\right\|_{(n+1) \times(n+1)}$ is transition probability matrix of irreducible Markov chains. If it arrives in the system $S_{j}$ message finds at least one service channel operable and free from the other messages it is immediately serviced and the time of service is a random variable with 
the parameter $\mu_{i}, i=\overline{1, n}$. Otherwise the message expects the beginning of service without restriction on duration of waiting. Let's assume that if the service channel would fail while completing some message, then after the restoration the interrupted message will be completed. Disciplines of the message processing in the network systems are FIFO. Assuming that the service time of messages, durations of serviceable work of channels and restoration time of service channels are independent random variables.

Our aim is to receive the system of the differential equations for the average number of messages and serviceable channels in the network QS at the large values of $K$. It should be noted that the presented techniques of the results reception has been offered for the first time in the works $[1,2]$ for the exponentional networks without the specified features (with reliable QS).

\section{The system of equations for the states probabilities}

The state of such a network at the moment $t$ could be described through vector

$$
z(t)=(d(t), k(t))=\left(d_{1}(t), d_{2}(t), \ldots, d_{n}(t), k_{1}(t), k_{2}(t), \ldots, k_{n}(t)\right),
$$

where $d_{i}(t)$ and $k_{i}(t)$ are the numbers of serviceable channels and the messages numbers in the system $S_{i}$ at the moment $t$ accordingly, $0 \leq d_{i}(t) \leq m_{i}$,

$0 \leq k_{i}(t) \leq K, t \in[0,+\infty), i=\overline{1, n}$. It is obvious that $k_{0}(t)=K-\sum_{i=1}^{n} k_{i}(t)-$ is the number of messages in the system $S_{0}$ at the moment $t$.

Vector $z(t)$ describes $2 n$-dimensional Markov process with the continuous time and the definite number of states. Let's consider, that

$$
P(d, k, t)=P(d(t)=d, k(t)=k),
$$

where $d=\left(d_{1}, d_{2}, \ldots, d_{n}\right), \quad 0 \leq d_{i} \leq m_{i}$ and $k=\left(k_{1}, k_{2}, \ldots, k_{n}\right), \quad 0 \leq k_{i} \leq K, \quad i=\overline{1, n}$. Let's denote $I_{i}$ as $n$-vector with zero components excluding $i$, that is equals to 1 . Let's describe the possible passages of Markov process $z(t)$ in the state $z(t+\Delta t)=(d, k, t+\Delta t)$ at the time $\Delta t:$

- from the state $(d, k, t)$ the passage is possible with probability

$$
1-\left[\mu_{0}\left(K-\sum_{i=1}^{n} k_{i}(t)\right)+\sum_{i=1}^{n}\left[\mu_{i} \min \left(m_{i}, k_{i}(t)\right)+\beta_{i} d_{i}(t)+\gamma_{i}\left(m_{i}-d_{i}(t)\right)\right]\right] \Delta t+o(t) \text {; }
$$


- from the state $\left(d, k-I_{i}, t\right)$ with probability

$$
\begin{gathered}
{\left[\mu_{0} p_{0 i}\left(K-\sum_{i=1}^{n} k_{i}(t)+1\right) \Delta t+o(\Delta t)\right]\left[1-\left\{\mu_{0} \sum_{\substack{j=1 \\
j \neq i}}^{n} p_{0 j}\left(K-k_{j}(t)\right)+\right.\right.} \\
\left.\left.+\sum_{j=1}^{n}\left[\mu_{j} \min \left(d_{j}(t), k_{j}(t)\right)+\beta_{j} d_{j}(t)+\gamma_{j}\left(m_{j}-d_{j}(t)\right)\right]\right\} \Delta t+o(\Delta t)\right], i=\overline{1, n} ;
\end{gathered}
$$

- from the state $\left(d, k+I_{i}, t\right)$ with probability

$$
\begin{gathered}
{\left[\mu_{i} p_{i 0} \min \left(d_{i}(t), k_{i}(t)+1\right) \Delta t+o(\Delta t)\right]\left[1-\left\{\mu_{0}\left(K-\sum_{j=1}^{n} k_{j}(t)+1\right)+\right.\right.} \\
+\sum_{\substack{j=1 \\
j \neq i}}^{n}\left[\mu_{j} \min \left(d_{j}(t), k_{j}(t)\right)+\beta_{j} d_{j}(t)+\gamma_{j}\left(m_{j}-d_{j}(t)\right)+\mu_{i} \min \left(d_{i}(t), k_{i}(t)\right)+\right. \\
\left.\left.+\beta_{i} d_{i}(t)+\gamma_{i}\left(m_{i}-d_{i}(t)\right)\right\} \Delta t+o(\Delta t)\right], i=\overline{1, n}
\end{gathered}
$$

- from the state $\left(d, k+I_{i}-I_{j}, t\right)$ with probability

$$
\begin{gathered}
{\left[\mu_{i} p_{i j} \min \left(d_{i}(t), k_{i}(t)+1\right) \Delta t+o(\Delta t)\right]\left[1-\left\{\mu_{0}\left(K-\sum_{j=1}^{n} k_{j}(t)\right)+\right.\right.} \\
+\sum_{\substack{r=1 \\
r \neq i, j}}^{n}\left[\mu_{r} \min \left(d_{r}(t), k_{r}(t)\right)+\beta_{r} d_{r}(t)+\gamma_{r}\left(m_{r}-d_{r}(t)\right)\right]+\mu_{i} \min \left(d_{i}(t), k_{i}(t)\right)+ \\
+\beta_{i} d_{i}(t)+\gamma_{i}\left(m_{i}-d_{i}(t)\right)+\mu_{j} \min \left(d_{j}(t), k_{j}(t)+1\right)+\beta_{j} d_{j}(t)+ \\
\left.\left.+\gamma_{j}\left(m_{j}-d_{j}(t)\right)\right\} \Delta t+o(\Delta t)\right], i, j=\overline{1, n}
\end{gathered}
$$

- from the state $\left(d-I_{i}, k, t\right)$ with probability

$$
\left[\gamma_{i}\left(m_{i}-d_{i}(t)+1\right) \Delta t+o(\Delta t)\right]\left[1-\left\{\mu_{0}\left(K-\sum_{j=1}^{n} k_{j}(t)\right)+\right.\right.
$$




$$
\left.\left.+\sum_{j=1}^{n}\left[\mu_{j} \min \left(d_{j}(t), k_{j}(t)\right)+\beta_{j} d_{j}(t)+\gamma_{j}\left(m_{j}-d_{j}(t)\right)\right]\right] \Delta t+o(\Delta t)\right\}, i=\overline{1, n}
$$

- from the state $\left(d+I_{i}, k, t\right)$ with probability

$$
\begin{gathered}
{\left[\beta_{i}\left(d_{i}(t)+1\right) \Delta t+o(\Delta t)\right]\left[1-\left\{\mu_{0}\left(K-\sum_{j=1}^{n} k_{j}(t)\right)+\right.\right.} \\
+\sum_{\substack{j=1 \\
j \neq i}}^{n}\left[\mu_{j} \min \left(d_{j}(t), k_{j}(t)\right)+\beta_{j} d_{j}(t)+\gamma_{j}\left(m_{j}-d_{j}(t)\right)\right]+\mu_{i} \min \left(d_{i}(t)+1, k_{i}(t)\right)+ \\
\left.\left.+\gamma_{i}\left(m_{i}-\left(d_{i}(t)+1\right)\right)\right] \Delta t+o(\Delta t)\right\}, i=\overline{1, n} ;
\end{gathered}
$$

- from all other states with probability $o(\Delta t)$.

Then, the usage of the formula of total probability makes it possible to write the system of difference equations for the states probabilities

$$
\begin{gathered}
P(d, k, t+\Delta t)=\sum_{i=1}^{n} \sum_{j=1}^{n} \mu_{i} p_{i j} \min \left(d_{i}(t), k_{i}(t)+1\right) P\left(d, k+I_{i}-I_{j}, t\right) \Delta t+ \\
+\mu_{0}\left(K-\sum_{i=1}^{n} k_{i}(t)+1\right) P\left(d, k-I_{j}, t\right) \Delta t+ \\
+\sum_{i=1}^{n} \mu_{i} p_{i 0} \min \left(d_{i}(t), k_{i}(t)+1\right) P\left(d, k+I_{i}, t\right) \Delta t+ \\
+\sum_{j=1}^{n} \gamma_{i}\left(m_{i}-d_{i}(t)+1\right) P\left(d-I_{j}, k, t\right) \Delta t+\sum_{i=1}^{n} \beta_{i}\left(d_{i}(t)+1\right) P\left(d+I_{i}, k, t\right) \Delta t+ \\
+\left\{1-\left[\mu_{0}\left(K-\sum_{i=1}^{n} k_{i}(t)\right)+\sum_{i=1}^{n} \mu_{i} p_{i 0} \min \left(d_{i}(t), k_{i}(t)\right)+\sum_{i=1}^{n} \gamma_{i}\left(m_{i}-d_{i}(t)\right)+\right.\right. \\
\left.\left.+\sum_{i=1}^{n} \beta_{i} d_{i}(t)\right] \Delta t\right\} P(d, k, t)+o(\Delta t),
\end{gathered}
$$

from which at $\Delta t \rightarrow 0$ we receive the system of difference-differential equations of Kolmogorov for the states probabilities 


$$
\begin{gathered}
\frac{d P(d, k, t)}{d t}=\sum_{i=1}^{n} \sum_{j=1}^{n} \mu_{i} p_{i j} \min \left(d_{i}(t), k_{i}\right)\left[P\left(d, k+I_{i}-I_{j}, t\right)-P(d, k, t)\right]+ \\
+\sum_{i=1}^{n} \sum_{j=1}^{n} \mu_{i} p_{i j}\left[\min \left(d_{i}(t), k_{i}(t)+1\right)-\min \left(d_{i}(t), k_{i}(t)\right)\right] P\left(d, k+I_{i}-I_{j}, t\right)+ \\
+\mu_{0}\left(K-\sum_{i=1}^{n} k_{i}(t)\right)\left[P\left(d, k-I_{j}, t\right)-P(d, k, t)\right]+\mu_{0} P\left(d, k-I_{j}, t\right)+ \\
+\sum_{i=1}^{n} \mu_{i} p_{i 0} \min \left(d_{i}(t), k_{i}(t)\right)\left[P\left(d, k+I_{i}, t\right)-P(d, k, t)\right]+ \\
+\sum_{i=1}^{n} \mu_{i} p_{i 0}\left[\min \left(d_{i}(t), k_{i}(t)+1\right)-\min \left(d_{i}(t), k_{i}(t)\right)\right] P\left(d, k+I_{i}, t\right)+ \\
+\sum_{j=1}^{n} \gamma_{i}\left(m_{i}-d_{i}(t)\right)\left[P\left(d-I_{j}, k, t\right)-P(d, k, t)\right]+\sum_{i=1}^{n} \gamma_{i} P\left(d-I_{i}, k, t\right)+ \\
+\sum_{i=1}^{n} \beta_{i} d_{i}(t)\left[P\left(d+I_{i}, k, t\right)-P(d, k, t)\right]+\sum_{i=1}^{n} \beta_{i} P\left(d+I_{i}, k, t\right) .
\end{gathered}
$$

The solution of this system in the analytical form is generally inconvenient. Therefore we will consider the important case of the large number of messages in the network, $K>>1$. In order to determine probability distribution of the random vector $z(t)$, it is convenient to switch to the relative variables, considering vector $\xi(t)=\left(\frac{d_{1}(t)}{K}, \frac{d_{2}(t)}{K}, \ldots, \frac{d_{n}(t)}{K}, \frac{k_{1}(t)}{K}, \frac{k_{2}(t)}{K}, \ldots, \frac{k_{n}(t)}{K}\right)$. In this case possible values of this vector at the fixed $t$ will belong to the bounded closed set

$$
G=\left\{(y, x)=\left(y_{1}, y_{2}, \ldots, y_{n}, x_{1}, x_{2}, \ldots, x_{n}\right): x_{i} \geq 0, \sum_{i=1}^{n} x_{i} \leq 1,0 \leq y_{i} \leq \frac{m_{i}}{K}\right\},
$$

in which they place in the nodes of the $2 n$-dimensional grid at the distance $\varepsilon=\frac{1}{K}$ from each other. While magnifying $K$ "the charging density" of set $G$ with the possible components of vector $\xi(t)$ will increase, and it is possible to consider, that it has a continuous distribution with the probabilities density $p(y, x, t)$, and $K^{2 n} P(d, k, t) \underset{K \rightarrow \infty}{\longrightarrow} p(y, x, t)$. Therefore it is possible to use the approximation of the function $P(d, k, t)$, using the relation $K^{2 n} P(d, k, t)=$ $=K^{2 n} P(y K, x K, t)=p(y, x, t),(y, x) \in G$. 
Let denote that $e_{i}=\varepsilon I_{i}, i=\overline{1, n}, c(b)=\left\{\begin{array}{l}1, b>0 \\ 0, b \leq 0\end{array}\right.$, and

$$
\min (b, a+1)=\min (b, a)+c(b-a), \quad c(b-a)=\frac{\partial \min (b, a)}{\partial a},
$$

thus $\min (b, a)=\left\{\begin{array}{l}a, b \geq a \\ b, b<a\end{array}\right.$. Using the relative variables $y_{i}=\frac{d_{i}}{K}, x_{i}=\frac{k_{i}}{K}, l_{i}=\frac{m_{i}}{K}$, $i=\overline{1, n}$, expression (4) and that if $K \rightarrow \infty, \varepsilon \rightarrow 0$, system (2) can be written as follows:

$$
\begin{gathered}
\frac{\partial p(y, x, t)}{\partial t}=\sum_{i=1}^{n} \sum_{j=1}^{n} K \mu_{i} p_{i j} \min \left(y_{i}, x_{i}\right)\left[p\left(y, x+e_{i}-e_{j}, t\right)-p(y, x, t)\right]+ \\
+\sum_{i=1}^{n} \sum_{j=1}^{n} \mu_{i} p_{i j} \frac{\partial \min \left(y_{i}, x_{i}\right)}{\partial x_{i}} p\left(y, x+e_{i}-e_{j}, t\right)+ \\
+K \mu_{0}\left(1-\sum_{i=1}^{n} x_{i}\right)\left[p\left(y, x-e_{j}, t\right)-p(y, x, t)\right]+\mu_{0}\left(p y, x-e_{j}, t\right)+ \\
+\sum_{i=1}^{n} K \mu_{i} p_{i 0} \min \left(y_{i}, x_{i}\right)\left[p\left(y, x+e_{i}, t\right)-(p y, x, t)\right]+ \\
+\sum_{i=1}^{n} \mu_{i} p_{i 0} \frac{\partial \min \left(y_{i}, x_{i}\right)}{\partial x_{i}} p\left(y, x+e_{i}, t\right)+ \\
+\sum_{i=1}^{n} K \gamma_{i}\left(l_{i}-y_{i}\right)\left[p\left(y-e_{i}, x, t\right)-p(y, x, t)\right]+\sum_{j=1}^{n} \gamma_{i} p\left(y-e_{i}, x, t\right)+ \\
+\sum_{i=1}^{n} K \beta_{i} y_{i}\left[p\left(y+e_{i}, x, t\right)-p(y, x, t)\right]+\sum_{i=1}^{n} \beta_{i} p\left(y+e_{i}, x, t\right) .
\end{gathered}
$$

\section{The system of differential equations for expected characteristics}

Let's present the right part (5) with the accuracy of term $\varepsilon^{2}$. If $p(y, x, t)$ is twice continuously differentiated at $y$ and $x$, than

$$
\begin{gathered}
p\left(y, x \pm e_{i}, t\right)=p(y, x, t) \pm \varepsilon \frac{\partial p(y, x, t)}{\partial x_{i}}+\frac{\varepsilon^{2}}{2} \frac{\partial^{2} p(y, x, t)}{\partial x_{i}^{2}}+o\left(\varepsilon^{2}\right) \\
p\left(y, x+e_{i}-e_{j}, t\right)=p(y, x, t)+\varepsilon\left(\frac{\partial p(y, x, t)}{\partial x_{i}}-\frac{\partial p(y, x, t)}{\partial x_{j}}\right)+
\end{gathered}
$$




$$
\begin{gathered}
+\frac{\varepsilon^{2}}{2}\left(\frac{\partial^{2} p(y, x, t)}{\partial x_{i}^{2}}-2 \frac{\partial^{2} p(y, x, t)}{\partial x_{i} \partial x_{j}}+\frac{\partial^{2} p(y, x, t)}{\partial x_{j}^{2}}\right)+o\left(\varepsilon^{2}\right), \\
p\left(y \pm e_{i}, x, t\right)=p(y, x, t) \pm \varepsilon \frac{\partial p(y, x, t)}{\partial y_{i}}+\frac{\varepsilon^{2}}{2} \frac{\partial^{2} p(y, x, t)}{\partial y_{i}^{2}}+o\left(\varepsilon^{2}\right), i=\overline{1, n} .
\end{gathered}
$$

Using them and that $\varepsilon K=1$, we receive

$$
\begin{aligned}
& \frac{\partial p(y, x, t)}{\partial t}=\sum_{i=1}^{n} \sum_{j=1}^{n} \mu_{i} p_{i j} \min \left(y_{i}, x_{i}\right)\left[\left(\frac{\partial p(y, x, t)}{\partial x_{i}}-\frac{\partial p(y, x, t)}{\partial x_{j}}\right)+\right.\left.\varepsilon\left(\frac{\partial^{2} p(y, x, t)}{\partial x_{i}^{2}}-2 \frac{\partial^{2} p(y, x, t)}{\partial x_{i} \partial x_{j}}+\frac{\partial^{2} p(y, x, t)}{\partial x_{j}^{2}}\right)\right]+ \\
&+ \frac{\partial}{2} \sum_{i=1}^{n} \sum_{j=1}^{n} \mu_{i} p_{i j} \frac{\partial \min \left(y_{i}, x_{i}\right)}{\partial x_{i}}\left[p(y, x, t)+\varepsilon\left(\frac{\partial p(y, x, t)}{\partial x_{i}}-\frac{\partial p(y, x, t)}{\partial x_{j}}\right)+\right. \\
&+\left.\frac{\varepsilon^{2}}{2}\left(\frac{\partial^{2} p(y, x, t)}{\partial x_{i}^{2}}-2 \frac{\partial^{2} p(y, x, t)}{\partial x_{i} \partial x_{j}}+\frac{\partial^{2} p(y, x, t)}{\partial x_{j}^{2}}\right)\right]+ \\
&+ \sum_{i=1}^{n} \mu_{0} p_{0 i}\left(1-\sum_{i=1}^{n} x_{i}\right)\left[-\frac{\partial p(y, x, t)}{\partial x_{i}}+\frac{\varepsilon}{2} \frac{\partial^{2} p(y, x, t)}{\partial x_{i}^{2}}\right]+ \\
&+ \mu_{0}\left[p(y, x, t)-\varepsilon \frac{\partial p(y, x, t)}{\partial x_{i}}+\frac{\varepsilon^{2}}{2} \frac{\partial^{2} p(y, x, t)}{\partial x_{i}^{2}}\right]+ \\
&+ \sum_{i=1}^{n} \mu_{i} p_{i 0} \min \left(y_{i}, x_{i}\right)\left[\frac{\partial p(y, x, t)}{\partial x_{i}}+\frac{\varepsilon}{2} \frac{\partial^{2} p(y, x, t)}{\partial x_{i}^{2}}\right]+ \\
& \frac{\partial \min \left(y_{i}, x_{i}\right)}{\partial x_{i}}\left[p(y, x, t)+\varepsilon \frac{\partial p(y, x, t)}{\partial x_{i}}+\frac{\varepsilon^{2}}{2} \frac{\partial^{2} p(y, x, t)}{\partial x_{i}^{2}}\right]+ \\
&++\sum_{i=1}^{n} \gamma_{i}\left(l_{i}-y_{i}\right)\left[-\frac{\partial p(y, x, t)}{\partial y_{i}}+\frac{\varepsilon}{2} \frac{\partial^{2} p(y, x, t)}{\partial y_{i}^{2}}\right]+ \\
&+\sum_{i=1}^{n} \mu_{i} p_{i 0} {\left[p(y, x, t)-\varepsilon \frac{\partial p(y, x, t)}{\partial y_{i}}+\frac{\varepsilon^{2}}{2} \frac{\partial^{2} p(y, x, t)}{\partial y_{i}^{2}}\right]+} \\
&+\beta_{i} y_{i}\left[\frac{\partial p(y, x, t)}{\partial y_{i}}+\frac{\varepsilon}{2} \frac{\partial^{2} p(y, x, t)}{\partial y_{i}^{2}}++\right. \\
&+
\end{aligned}
$$




$$
+\sum_{i=1}^{n} \beta_{i}\left[p(y, x, t)+\varepsilon \frac{\partial p(y, x, t)}{\partial y_{i}}+\frac{\varepsilon^{2}}{2} \frac{\partial^{2} p(y, x, t)}{\partial y_{i}^{2}}\right]+o\left(\varepsilon^{2}\right) .
$$

Thus, the density $p(y, x, t)$ is satisfied with the accuracy within the term $\varepsilon^{2}$ to the Kolmogorov-Fokker-Planc equation:

$$
\begin{gathered}
\frac{\partial p(y, x, t)}{\partial t}=-\sum_{i=1}^{n} \frac{\partial}{\partial y_{i}}\left(A_{i}^{(1)}(y) p(y, x, t)\right)-\sum_{i=1}^{n} \frac{\partial}{\partial x_{i}}\left(A_{i}^{(2)}(y, x) p(y, x, t)\right)+ \\
+\frac{\varepsilon}{2} \sum_{i, j=1}^{n} \frac{\partial^{2}}{\partial y_{i} \partial y_{j}}\left(B_{i j}^{(1)}(y) p(y, x, t)\right)+\frac{\varepsilon}{2} \sum_{i, j=1}^{n} \frac{\partial^{2}}{\partial x_{i} \partial x_{j}}\left(B_{i j}^{(2)}(y, x) p(y, x, t)\right),
\end{gathered}
$$

where

$$
\begin{gathered}
A_{i}^{(1)}(y)=\gamma_{i}\left(l_{i}-y_{i}\right)-\beta_{i} y_{i}, \\
A_{i}^{(2)}(y, x)=\sum_{j=1}^{n} \mu_{j} p_{j i}^{*} \min \left(y_{j}, x_{j}\right)+\mu_{0} p_{0 i}\left(1-\sum_{i=1}^{n} x_{i}\right), i=\overline{1, n}, \\
p_{j i}^{*}=\left\{\begin{array}{l}
p_{j i}, j \neq i, \quad B_{i i}^{(1)}(y)=\gamma_{i}\left(l_{i}-y_{i}\right)+\beta_{i} y_{i} ; \quad B_{i j}^{(1)}(y)=0, \quad i \neq j ; \\
p_{i i}-1, j=i ;
\end{array}\right. \\
B_{i i}^{(2)}(y, x)=\sum_{j=1}^{n} \mu_{j} p_{j i}^{* *} \min \left(y_{j}, x_{j}\right)+\mu_{0} p_{0 i}\left(1-\sum_{i=1}^{n} x_{i}\right), \\
p_{j i}^{* *}=\left\{\begin{array}{l}
p_{j i}, j \neq i, \\
1-p_{i i}, j=i ;
\end{array} \quad B_{i j}^{(2)}(y, x)=-\mu_{i} p_{i j} \min \left(y_{i}, x_{i}\right), \quad i \neq j, i=\overline{1, n} .\right.
\end{gathered}
$$

As the density $p(y, x, t)$ satisfies the Kolmogorov-Fokker-Planc equation and $A_{i}^{(1)}(y), A_{i}^{(2)}(y, x)$ piecewise linear functions on $y, x$, according to [3], the mathematical expectations $w_{i}(t)=M\left\{\frac{d_{i}(t)}{K}\right\}, \quad n_{i}(t)=M\left\{\frac{k_{i}(t)}{K}\right\}, \quad i=\overline{1, n}$, with the accuracy within the terms of infinitesimal order $O\left(\varepsilon^{2}\right)$ are defined from the systems of the equations

$$
\frac{d w_{i}(t)}{d t}=A_{i}^{(1)}(w(t))=\gamma_{i}\left(l_{i}-w_{i}(t)\right)-\beta_{i} w_{i}(t), i=\overline{1, n},
$$




$$
\frac{d n_{i}(t)}{d t}=A_{i}^{(2)}(w(t), n(t))=\sum_{j=1}^{n} \mu_{j} p_{j i}^{*} \min \left(w_{j}(t), n_{j}(t)\right)+\mu_{0} p_{0 i}\left(1-\sum_{i=1}^{n} n_{i}(t)\right),
$$

The right hand sides of system (11) are continuous piecewise linear functions. By segmentation of phase space and obtaining solutions of system (11) in ranges of right hand sides linearity it is possible to solve the whole system.

Let $\Omega(t)=\{1,2, \ldots, n\}$ - be set of vector $n(t)$ component indices. Let's divide $\Omega(t)$ into two disjoint sets $\Omega_{0}(t)$ and $\Omega_{1}(t)$ :

$$
\Omega_{0}(t)=\left\{i: w_{i}(t)<n_{i}(t) \leq 1\right\}, \quad \Omega_{1}(t)=\left\{j: 0 \leq n_{j}(t) \leq w_{j}(t)\right\} .
$$

Each partitioning specifies disjoint regions $G_{\tau}(t)$ in the set

$$
G(t)=\left\{n(t): n_{i}(t) \geq 0, \sum_{i=1}^{n} n_{i}(t) \leq 1\right\}
$$

such that:

$$
\begin{gathered}
G_{\tau}(t)=\left\{n(t): w_{i}(t)<n_{i}(t) \leq 1, i \in \Omega_{0}(t) ;\right. \\
\left.0 \leq n_{j}(t) \leq w_{j}(t), j \in \Omega_{1}(t) ; \sum_{c=1}^{n} n_{c}(t) \leq 1\right\}, \quad \tau=1,2, \ldots, 2^{n}, \bigcup_{\tau=1}^{2^{n}} G_{\tau}(t)=G(t) .
\end{gathered}
$$

Then system of equations (11) of explicit form for each region $G_{\tau}(t)$ is:

$$
\frac{d n_{i}(t)}{d t}=\sum_{0} \mu_{j} p_{j i}^{*} w_{j}(t)+\sum_{1} \mu_{j} p_{j i}^{*} n_{j}(t)+\mu_{0} p_{0 i}\left(1-\sum_{i=1}^{n} n_{i}(t)\right), i=\overline{1, n},
$$

where $\sum_{0}=\sum_{j \in \Omega_{0}(t)}, \quad \sum_{1}=\sum_{j \in \Omega_{1}(t)}$. The solution of the system of equations (10), (12) makes it possible to obtain an average relative number of messages and serviceable channels at any QS of queueing network. At the region $A: \Omega_{0}(t)=\{\varnothing\}$, $\Omega_{1}(t)=\{1,2, \ldots, n\}$, when queues in average at the system are absent, systems (10), (12) look like

$$
\begin{gathered}
\frac{d w_{i}(t)}{d t}=\gamma_{i}\left(l_{i}-w_{i}(t)\right)-\beta_{i} w_{i}(t)=\gamma_{i} l_{i}-\left(\gamma_{i}+\beta_{i}\right) w_{i}(t), \\
\frac{d n_{i}(t)}{d t}=\sum_{1} \mu_{j} p_{j i}^{*} n_{j}(t)+\mu_{0} p_{0 i}\left(1-\sum_{i=1}^{n} n_{i}(t)\right) .
\end{gathered}
$$




\section{Example}

Let's consider the closed exponential queueing network with unreliable service channels and central QS, $n=4, K=1000 ; m_{1}=11, m_{2}=9, m_{3}=10, m_{4}=15$. The intensity of arrival of messages from outside medium (system $S_{0}$ ) is $\mu_{0}=5$. Intensity of service of messages in service channels of QS are $\mu_{1}=1.5, \mu_{2}=1$, $\mu_{3}=1.4, \mu_{4}=0.9$. Averages of duration of serviceable work for each service channel of QS $\beta_{1}^{-1}=1.65, \beta_{2}^{-1}=1.41, \beta_{3}^{-1}=1.4, \beta_{4}^{-1}=1.31$ are equal accordingly. Averages of duration of reconstruction of faulty service channels of QS are $\gamma_{1}^{-1}=1.31, \gamma_{2}^{-1}=0.9, \gamma_{3}^{-1}=1.2, \gamma_{4}^{-1}=1.65$. Probabilities of transition of messages: $p_{01}=p_{02}=p_{03}=p_{04}=p_{10}=p_{20}=p_{30}=p_{40}=1 / 4, \quad p_{14}=p_{24}=p_{34}=3 / 4, \quad p_{14}=1 / 2$, $p_{42}=p_{43}=1 / 8$, other $p_{i j}=0, i, j=\overline{0,4}$. At the initial moment of time $t=0$ : $n_{i}(0)=0, w_{i}(0)=m_{i}$, and $\min \left(w_{i}(t), n_{i}(t)\right)=n_{i}(t), i=\overline{1,4}$. Then the system (14) is a system of non-homogeneous linear differential equations and can be written in the vector form

$$
\frac{d n(t)}{d t}=A n(t)+Q(t)
$$

where $n^{T}(t)=\left(n_{1}(t), n_{2}(t), \ldots, n_{5}(t),\right)$ - vector of average relative number of messages in each of QS. The decision of system (14) can be found as

$$
n(t)=e^{A t} n(0)+\int_{0}^{t} e^{A(t-\tau)} Q(\tau) d \tau .
$$

Analytical expressions for change of average relative number of messages in network systems look like

$$
\begin{gathered}
n_{1}(t)=0.002+0.0031 e^{-1.9998 t}-0.003 e^{-5.3195 t}-0.0017 e^{-1.4196 t}+0.0005 e^{-1.0609 t}, \\
n_{2}(t)=0.0012+0.0002 e^{-1.9998 t}-0.0027 e^{-5.3195 t}+0.0001 e^{-1.4196 t}+0.0011 e^{-1.0609 t}, \\
n_{3}(t)=0.0009+0.0004 e^{-1.9998 t}-0.0003 e^{-5.3195 t}+0.0019 e^{-1.4196 t}+0.0002 e^{-1.0609 t}, \\
n_{4}(t)=0.0053-0.0039 e^{-1.9998 t}-0.0007 e^{-5.3195 t}-0.0003 e^{-1.4196 t}-0.0004 e^{-1.0609 t},
\end{gathered}
$$

When these expressions are multiplied on $K$ we will receive expressions for an average of messages in QS $N_{i}(t)=K n_{i}(t), i=\overline{1,4}$. Figures of change $N_{1}(t)$, $N_{3}(t)$ are presented in Figures 1 and 2. 


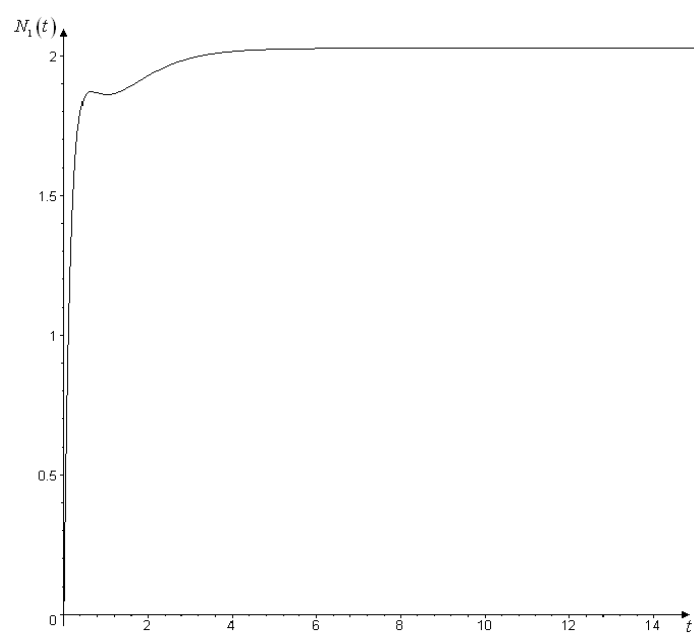

Fig. 1. Change of average number of messages in QS $S_{1}$

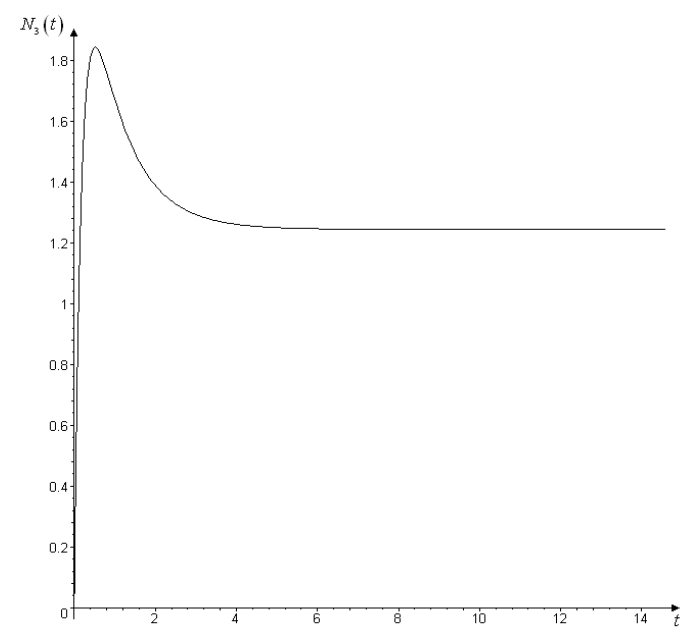

Fig. 2. Change of average number of messages in QS $S_{3}$

Solving system (13) we will receive expressions for the average relative number of serviceable channels in network systems:

$$
\begin{aligned}
& w_{1}(t)=0.0061+0.0049 e^{-1.3694 t}, w_{2}(t)=0.0055+0.0035 e^{-1.8203 t}, \\
& w_{3}(t)=0.0054+0.0046 e^{-1.5476 t}, w_{4}(t)=0.0066+0.0084 e^{-1.3694 t} .
\end{aligned}
$$

from $d_{i}(t)=K w_{i}(t), i=\overline{1,4}$. Figures of change $d_{1}(t), d_{3}(t)$ are presented in Figures 3 and 4 . 


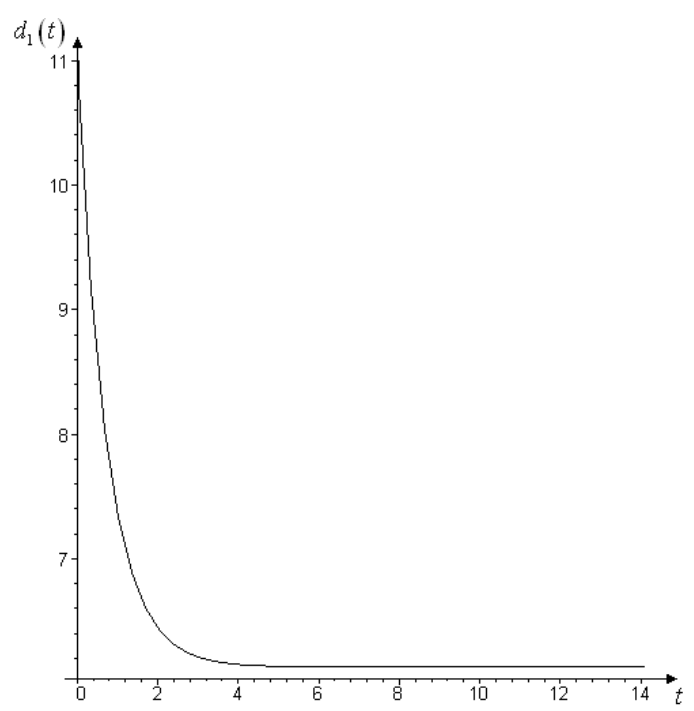

Fig. 3. Change of average number of serviceable channels in QS $S_{1}$

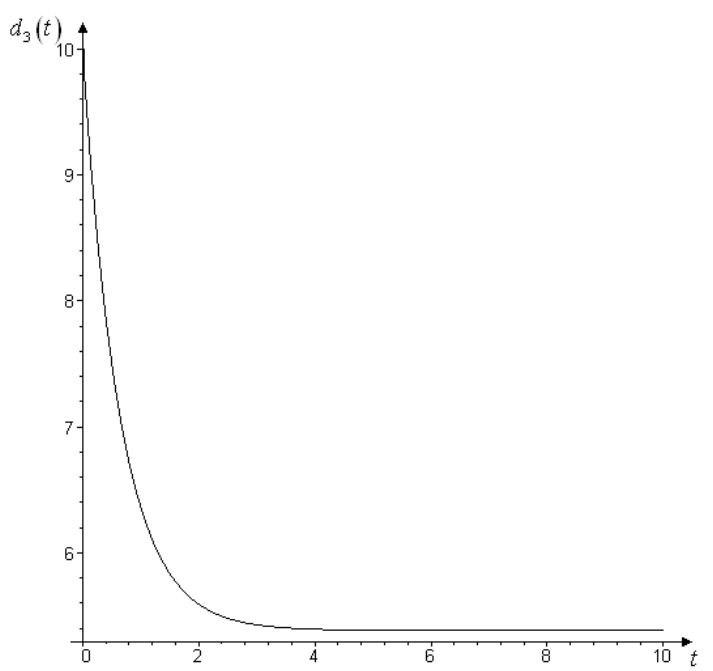

Fig. 4. Change of average number of serviceable channels in QS $S_{3}$

\section{Conclusions}

The considered method of diffusive approximations for finding the average number of messages and serviceable channels of QN makes it possible to precisely define of the given average characteristics in the stationary and transient regime. The accuracy of method increases with an increase in a number of messages in the network. 


\section{References}

[1] Medvedev G.A., Closed queueing systems and their optimization, Proceedings of the USSR Academy of Sciences: Engineering Cybernetics, 1978, 6, 199-203 (in Russian).

[2] Medvedev G.A., About optimization of closed queueing systems, Proceedings of the USSR Academy of Sciences: Engineering Cybernetics, 1975, 6, 65-73 (in Russian).

[3] Parajev Y.I., Introduction Statistical Dynamics of Control Processes, Sov. Radio, Moskow 1976 (in Russian). 